\title{
Análise das intervenções e dos fatores socioambientais associados à ocorrência de tracoma em Pernambuco a partir de dois inquéritos em escolares realizados em 2006 e 2012
}

\author{
Analysis of interventions and socio environmental factors \\ associated with the occurrence of trachoma in Pernambuco in two \\ surveys on schoolchildren conducted in 2006 and 2012
}

\author{
Fabia Alexandra Pottes Alves ${ }^{1}$, Wayner Vieira de Souza², Carlos Feitosa Luna², \\ Giselle Campozana Gouveia²
}

\begin{abstract}
Resumo
O tracoma é a principal causa de cegueira prevenível nos países em desenvolvimento. O objetivo foi descrever o panorama do tracoma entre escolares no Estado de Pernambuco, identificando as estratégias norteadoras do programa de controle e analisando a existência de associação entre variáveis socioeconômicas, demográficas e de saúde, em âmbito ecológico, com as taxas de prevalência da doença obtidas nos inquéritos realizados em 2006 e 2012. Realizou-se um estudo ecológico descritivo, com dados secundários de inquéritos realizados em escolas públicas de 79 municípios de Pernambuco, em 2006, e de 22 municípios, em 2012. O pequeno número de examinados em 2006 resultou em estimativas imprecisas de prevalência, fragilizando metodologicamente a escolha dos 22 municípios considerados prioritários pelo Programa SANAR em 2012. Só foi possível comprovar a diminuição da prevalência do tracoma apenas em 5 dos 22 prioritários investigados. Investigações de base populacional deverão ser executadas no Estado e as ações de controle deverão ser repensadas, pois o formato das investigações ainda é baseado em campanhas com ações pontuais, sem o desenvolvimento de outras contínuas e efetivas que reduzam as formas ativas da doença, alcançando, assim, a meta de eliminação do tracoma com causa de cegueira no Brasil.
\end{abstract}

Palavras-chave: tracoma; pré-escolar; epidemiologia.

\begin{abstract}
Trachoma is the main cause of preventable blindness in developing countries. This paper aims to describe the trachoma situation in the schools of Pernambuco, Brazil, noting guidelines for the control program and analyzing a possible association of the prevalence rates obtained by research in 2006 and 2012 with socioeconomic, demographic and ecological health factors. A descriptive ecological study was performed, using secondary data from investigations within Pernambuco public schools (investigating 79 cities in 2006 and 22 in 2012). Due to the small number of patients examined in 2006, the prevalence numbers estimative was rather inaccurate, methodologically impairing the choice of the 22 cities considered priorities for the SANAR programme in 2012. However, it was only possible to confirm the reduction of Trachoma prevalence in 5 among the 22 priority cities investigated. Demographic studies shall be done and the containing measures shall be reviewed in the state, since the investigation layout is still based in punctual actions campaigns, without the development of other continuous and effective measures that reduce the active types of the disease in order to eliminate Trachoma as a cause of blindness in Brazil.
\end{abstract}

Keywords: trachoma; preschool; epidemiology.

${ }^{1}$ Universidade Federal de Pernambuco (UFPE) - Recife (PE), Brasil.

${ }^{2}$ Departamento de Saúde Coletiva, Centro de Pesquisas Aggeu Magalhães (CPQAM), Fundação Oswaldo Cruz (FIOCRUZ) - Recife (PE), Brasil.

Trabalho realizado no Centro de Pesquisas Aggeu Magalhães (CPQAM), Fundação Oswaldo Cruz (FIOCRUZ) - Recife (PE), Brasil.

Endereço para correspondência: Fabia Alexandra Pottes Alves - Universidade Federal de Pernambuco, Rua do Espinheiro, 685/102 - Espinheiro - CEP: 52020-

020 - Recife (PE), Brasil - Email: fabia.alexandra@terra.com.br

Fonte de financiamento: nenhuma.

Conflito de interesses: nada a declarar.

Cad. Saúde Colet., 2016, Rio de Janeiro, 24 (4): 435-442 


\section{INTRODUÇÃO}

O tracoma é uma ceratoconjuntivite bacteriana crônica recidivante, causada pela Chlamydia trachomatis (sorotipos $\mathrm{A}, \mathrm{B}, \mathrm{Ba}$ e C) e que costuma afetar crianças desde os primeiros meses de vida, desenvolvendo-se de forma lenta. Em decorrência de infecções repetidas, são produzidas cicatrizes na conjuntiva palpebral superior, podendo levar à formação de entrópio (pálpebra com a margem virada para dentro do olho), triquíase (cílios invertidos), opacidade corneana, olho seco e cegueira no adulto ${ }^{1}$.

A única fonte de infecção é o homem com a doença ativa na conjuntiva ou em outras mucosas. O modo de transmissão pode ser direto, pessoa a pessoa, ou indireto, por meio de compartilhamento de objetos contaminados, tais como toalhas, lenços, roupas de cama de uso comum, além de também ser transmitida por vetores mecânicos, a exemplo de insetos como a mosca doméstica ${ }^{1}$. Geralmente sua transmissão ocorre dentro do ambiente doméstico quando existem lesões ativas da doença.

As formas inflamatórias do tracoma predominam em crianças entre 1 e 10 anos, ocorrendo com maior frequência onde há falta de água e condições habitacionais e sanitárias inadequadas ${ }^{1-4}$.

A Organização Mundial de Saúde (OMS) estima que o tracoma afete 21,4 milhões de pessoas, das quais 7,6 milhões estejam com triquíase tracomatosa (TT), enquanto 5 milhões estejam com sérios prejuízos visuais e cegueira devido às complicações da doença. Portanto, trata-se da principal causa de cegueira prevenível, principalmente nos países em desenvolvimento ${ }^{5,6}$.

Historicamente, o tracoma chegou ao Brasil no século XVIII trazido pelos ciganos expulsos de Portugal, desenvolvendo-se na região do Cariri, interior do Ceará, e expandindo-se para toda a região Nordeste. Outros dois focos importantes surgiram nos Estados de São Paulo e Rio Grande do Sul, trazidos pelos imigrantes italianos e espanhóis, tendo sido decisivos na disseminação da doença no restante do país ${ }^{1}$.

O tracoma é considerado uma doença tropical negligenciada (DTN) e, segundo a OMS, faz parte de um conjunto heterogêneo de doenças causadas por agentes infecciosos ou parasitários, provocando um grande impacto sobre a saúde e o bem-estar da população atingida ${ }^{6}$. Essas doenças são assim denominadas porque se encontram fora da agenda de pesquisa e desenvolvimento das grandes indústrias farmacêuticas ou do investimento dos países desenvolvidos. Sua perpetuação contribui para a manutenção dos ciclos de pobreza, desigualdade e exclusão social em razão, principalmente, de seu impacto na saúde infantil, na redução da produtividade da população trabalhadora e na promoção do estigma social ${ }^{7}$.

A partir de 1997, o tracoma foi reconhecido como um problema de saúde pública, quando foi lançada a Aliança para Eliminação Global do Tracoma até 2020 (GET2020), que visava à eliminação de toda a cegueira evitável ${ }^{5}$. Os indicadores epidemiológicos para obtenção, com a OMS, da certificação de eliminação do tracoma como causa de cegueira são: ter menos de um caso de TT por 1.000 habitantes e menos de 5\% de tracoma inflamatório folicular (TF) em crianças menores de 10 anos. Para o alcance desse objetivo, foi lançada a estratégia SAFE, sigla em inglês que quer dizer: (S) Cirurgia dos casos de TT; (A) Antibioticoterapia nos casos de tracoma ativo; (F) Higiene facial/educação em saúde; (E) Melhoria do meio ambiente/saneamento básico e ampliação do acesso à água, devendo ser implementada nos países endêmicos ${ }^{7,8}$.

O Brasil faz parte dessa aliança internacional e assumiu esse compromisso para a eliminação do tracoma como causa de cegueira até o ano de 2015. Por essa razão, vem desenvolvendo um trabalho com os coordenadores estaduais dos programas de vigilância e controle do tracoma, visando à efetivação do plano de eliminação do tracoma como causa de cegueira.

Com o objetivo de conhecer a real situação do tracoma no país, o Ministério da Saúde (MS) desenvolveu, no período de 2002 a 2007, um inquérito epidemiológico de tracoma em escolares em 18 Estados e no Distrito Federal para investigar a ocorrência e distribuição dessa doença. Foram examinados 119.531 alunos, distribuídos em 2.270 escolas, localizadas em 1.156 municípios, selecionados por terem Índice de Desenvolvimento Humano Municipal (IDH-M) menor que a média nacional ${ }^{9}$. A etapa referente ao Estado de Pernambuco aconteceu no ano de 2006, quando foram investigados 79 municípios.

Em 2011, o MS lançou o plano integrado de ações estratégicas de eliminação do tracoma como causa de cegueira e de outras DTN. A proposta tinha como estratégia de ação primordial a intensificação das práticas de busca ativa de casos em sua forma inflamatória transmissível - TF -, em crianças de 1 a 9 anos de idade de comunidades locais e a busca ativa de casos do tracoma em sua forma sequelar $-\mathrm{TT}^{8}$. Em Pernambuco, a Secretaria Estadual de Saúde (SES) criou, naquele mesmo ano, um plano para redução e eliminação das doenças negligenciadas no Estado, chamado de "Programa SANAR", que elegeu sete agravos prioritários entre as DTN mais persistentes no Estado, dentre elas o tracoma. Para alcançar a meta de redução do tracoma preconizada pela OMS, o Programa SANAR selecionou 22 municípios do Estado, onde implementou ações para a redução da prevalência da doença até $2014^{10}$. Diante desse contexto, este estudo tem como objetivo descrever o panorama do tracoma entre escolares no Estado de Pernambuco, identificando as estratégias norteadoras do programa de controle e analisando a existência de associação entre variáveis socioeconômicas, demográficas e de saúde, em âmbito ecológico, com as taxas de prevalência da doença obtidas nos inquéritos realizados em 2006 e 2012. 


\section{MÉTODO}

Trata-se de um estudo ecológico cujas unidades de análise foram os 79 municípios de Pernambuco (Figura 1) avaliados na etapa estadual do inquérito nacional do tracoma, realizada em 2006 (em cinza-escuro), e em 22 municípios (em cinza-claro), selecionados dentre os 79 , segundo o inquérito conduzido pelo Programa SANAR, no ano de 2012.

No primeiro inquérito (2006), foram examinados 7.059 alunos. Já no segundo, realizado em 2012, foram investigados 78.118 alunos de 1 a 15 anos. O critério utilizado na seleção dos municípios em Pernambuco no inquérito nacional, em 2006, foi investigar os que tivessem IDH-M inferior à média nacional, com base no ano de 2000, que à época era de 0,764. Para a seleção dos alunos investigados, o critério foi estar matriculado da $1^{\text {a a }} 4^{\text {a }}$ série do ensino fundamental nas escolas da rede pública. Não houve restrição quanto à idade 9 . Para a seleção dos municípios em 2012, o critério adotado foi de apresentar prevalência pontual de tracoma no inquérito anterior igual ou maior que 5\%. Para a seleção dos alunos investigados, o critério utilizado foi estudar na rede pública e estar na faixa etária de 1 a 15 anos no período.

Foram utilizados dados secundários oriundos dos dois inquéritos escolares, fornecidos mediante carta de anuência da SES. Os indicadores socioeconômicos, demográficos e de saúde foram retirados das bases de dados do Instituto Brasileiro de Geografia e Estatística (IBGE), do Departamento de Informática do Sistema Único de Saúde (DATASUS), do Atlas e Desenvolvimento Humano no Brasil do Programa das Nações Unidas para o Desenvolvimento (PNUD/2014) e da Secretaria de Atenção a Saúde (SAS)/Departamento de Atenção Básica (DAB), todas de domínio público. A variável dependente foi a prevalência de tracoma, e as variáveis independentes foram: densidade demográfica (2010); população residente até 15 anos (2010); produto interno bruto (PIB) per capita (2010); índice de Gini (2010); frequência líquida de alunos na pré-escola (2010); frequência líquida de alunos no ensino fundamental (2010); frequência líquida de alunos no ensino médio (2010); percentual de crianças extremamente pobres (2010); percentual da população em domicílios com banheiro e água encanada (2010); percentual da população em domicílios com coleta de lixo (2010); percentual de pessoas em domicílios com abastecimento de água e esgotamento sanitário inadequados (2010); percentual da população em domicílios com densidade maior que duas pessoas por dormitório (2010); percentual de cobertura da Estratégia Saúde da Família (2011).

$\mathrm{Na}$ análise, foram utilizadas as prevalências de tracoma dos dois inquéritos, nos quais foram considerados municípios prioritários os 22 selecionados em 2012 e não prioritários os 57 restantes, que fizeram parte do inquérito de 2006. Para analisar a evolução das prevalências de 2006 para 2012, foram construídos os respectivos intervalos de confiança (IC95\%) para o parâmetro do município em cada período, comparando-os.

Foi realizada uma análise descritiva dos indicadores sociodemográficos e de saúde a fim de identificar diferenças entre os municípios prioritários e os demais. Para comparar os indicadores socioeconômicos e de saúde dos municípios prioritários e não prioritários, foi aplicado o teste não paramétrico de Mann-Whitney.

Para comparar as prevalências de tracoma entre as áreas urbana e rural, foi aplicado o teste qui-quadrado de Pearson. E a fim de verificar a existência de associação entre os indicadores sociodemográficos e de saúde com a prevalência de tracoma no conjunto de municípios prioritários do Programa SANAR, foi calculado o coeficiente de correlação linear de Pearson.

O projeto foi aprovado pelo Comitê de Ética em Pesquisas com Seres Humanos do Centro de Pesquisas Aggeu Magalhães/FIOCRUZ, sob Parecer no 27/2013.

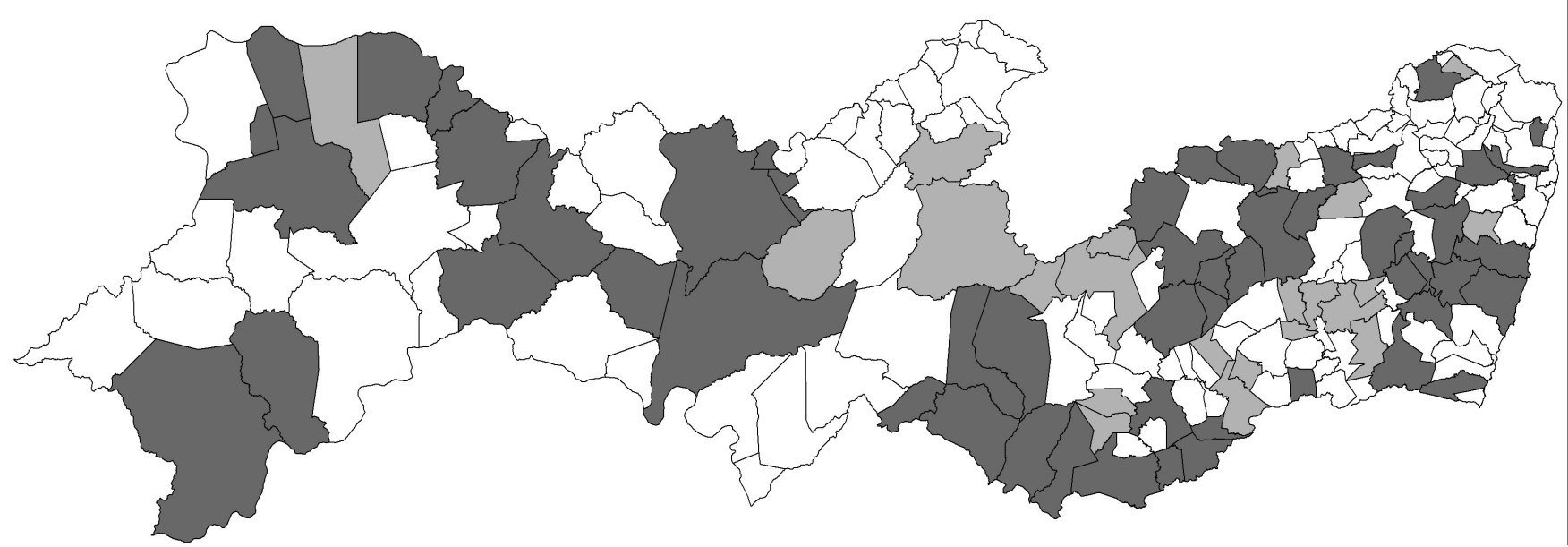

Figura 1. Mapa do Estado de Pernambuco com os municípios selecionados para os inquéritos de tracoma realizados em 2006 e 2012. Legenda: Cinza-escuro: municípios 2006; cinza-claro: municípios 2012 


\section{RESULTADOS}

Na etapa de Pernambuco do inquérito nacional do tracoma realizado em 2006, foram selecionados 79 dos 185 municípios do Estado, nos quais foram detectados 226 casos de tracoma, implicando em uma prevalência de 3,2\% (IC:2,8-3,6\%) no Estado. As prevalências variaram entre 0 e $17,7 \%$, tendo 22 desses municípios apresentado prevalências pontuais próximas ou superiores a $5 \%$, sendo assim considerados prioritários para o SANAR. No entanto, ao observarem-se os IC das prevalências nesses municípios, percebe-se bastante variabilidade nas estimativas devido ao pequeno número de examinados (total=7.059; municípios: mínimo $=35 ;$ p25=62; mediana $=79$; p75=109; máximo $=285$ ), revelando que para apenas 14 municípios pode-se afirmar que suas prevalências eram significativamente inferiores a 5\%. Dessa forma, 43 municípios deixaram de ser incluídos no rol de prioridades para o controle da endemia em Pernambuco (Figura 2).

No inquérito escolar realizado em 2012, foram identificados 2.306 casos de tracoma, com prevalência geral de 2,9\% (IC:2,8-3,1\%). Nesse inquérito, o número de examinados por município foi significativamente maior, permitindo estimativas mais precisas (Tabela 1 e Figura 2). Verificou-se que, em sete municípios, não se pode rejeitar a hipótese de que suas prevalências sejam maiores que $5 \%$. No entanto, ao comparar os IC das prevalências dos dois inquéritos, os dados revelam que apenas em cinco municípios os IC não se sobrepuseram, sugerindo que houve redução da prevalência do tracoma de 2006 para 2012 (Figura 2).

Ao analisar possíveis diferenças nos indicadores socioeconômicos, demográficos e de saúde dos municípios prioritários e não prioritários para o tracoma, identificou-se que os dois grupos de municípios possuem características bastante semelhantes, havendo diferença estatisticamente significante apenas no percentual da população que vive em domicílios com densidade maior que duas pessoas por dormitório - de forma aparentemente paradoxal, esse resultado foi maior nos municípios não prioritários $(\mathrm{p}=0,03)$ (Tabela 2$)$.

Na Tabela 1 apresenta-se a prevalência do tracoma segundo a zona de residência. Destacam-se as prevalências nos municípios de Barra de Guabiraba (9,1\%; IC:7,8-10,4\%) e São Joaquim do Monte (0,8\%; IC:0,5-1,3\%), que apresentaram maior e menor prevalência, respectivamente. Quanto à zona de residência dos casos, identificou-se que houve diferenças significantes das prevalências entre as zonas rural e urbana apenas em cinco municípios, porém deve-se destacar novamente o município de Barra de Guabiraba, que apresentou na zona rural uma prevalência de 12,9\% (IC:6-23\%), podendo estar acima do valor de referência para tratamento em massa, que é de $10 \%{ }^{1,8}$.

Buscando identificar os fatores associados às prevalências de tracoma nos municípios prioritários, foram calculados
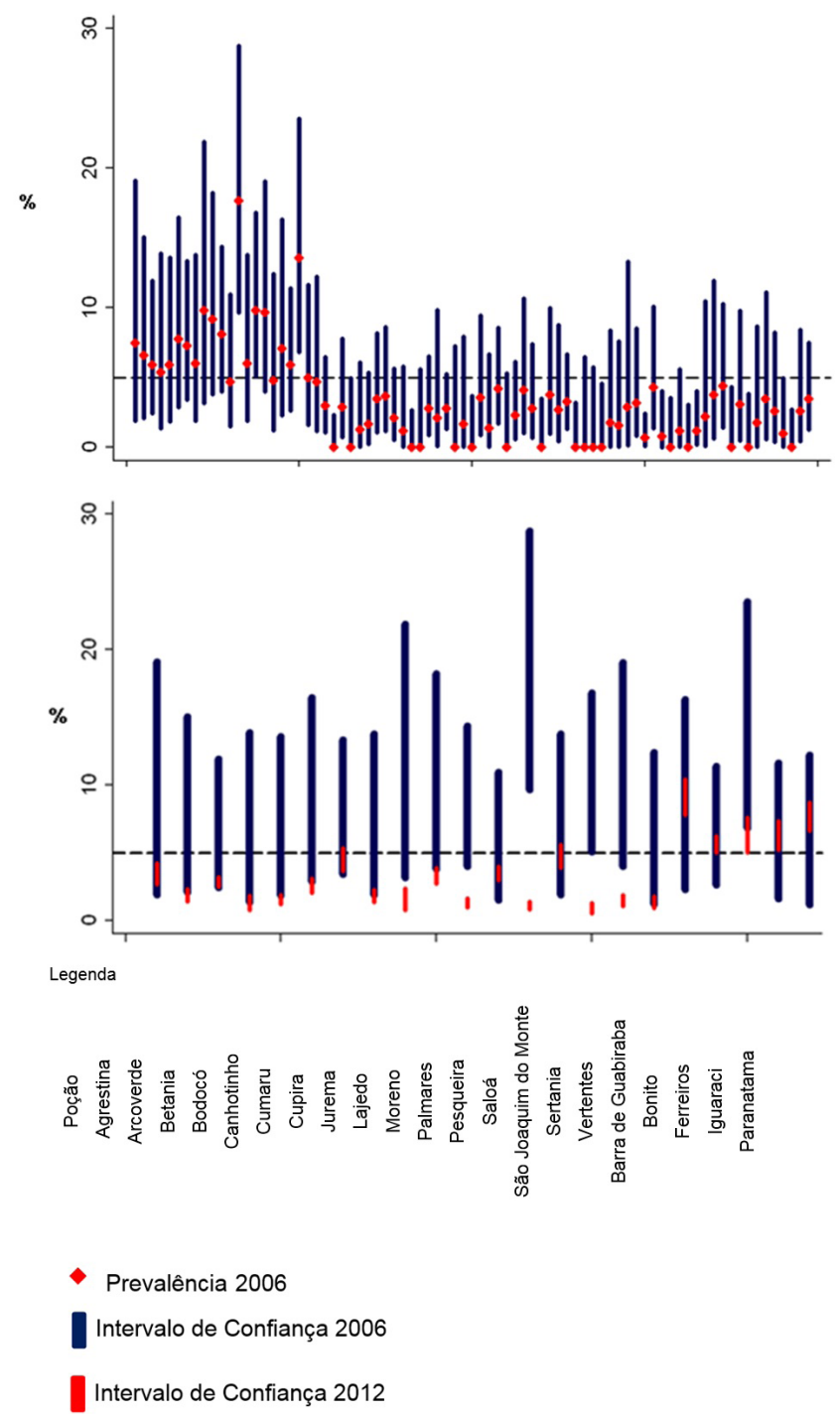

Figura 2. Prevalência e intervalo de confiança dos 79 municípios investigados na etapa estadual do inquérito escolar nacional do tracoma em 2006 e prevalência e intervalo de confiança dos 22 municípios prioritários para o tracoma, segundo os inquéritos escolares realizados em 2006 e 2012, em Pernambuco, Brasil. Fonte: Elaborado pela autora a partir dos dados da Secretaria Estadual de Saúde de Pernambuco (2006)

os coeficientes de correlação linear de Pearson ente essas prevalências e os indicadores socioambientais, com respectivos níveis de significância. Os resultados estão apresentados na Tabela 3, na qual se verifica que não há associação significante entre a prevalência de tracoma e qualquer dos indicadores socioeconômicos, demográficos e de saúde, refletindo uma homogeneidade nas condições de vida nesses municípios.

\section{DISCUSSÃO}

Avaliando o critério de seleção dos municípios de Pernambuco que fizeram parte do inquérito escolar nacional do tracoma em 2006, primeiramente pode-se questionar o ponto de corte adotado para o Estado (IDH-M abaixo da média nacional), além de registrar que vários municípios com IDH-M abaixo desse 
valor não integraram a lista dos 79 municípios pesquisados - existiam à época 180 nessa condição em Pernambuco. Tal abordagem, assim como a seleção apenas de escolares, não confere à investigação um caráter representativo da população em geral.
Em relação às prevalências identificadas nos municípios selecionados, observa-se que os IC apresentaram uma grande amplitude devido à pequena amostra de alunos avaliados em cada um desses municípios, revelando uma imprecisão nessas estimativas e causando uma fragilidade nos resultados apresentados.

Tabela 1. Distribuição dos 22 municípios prioritários para o tracoma em relação ao número de examinados, casos, prevalência, zona de residência e p-valor, em Pernambuco, Brasil, 2012

\begin{tabular}{|c|c|c|c|c|c|c|c|c|c|c|}
\hline \multirow{2}{*}{ Municípios } & \multicolumn{3}{|c|}{ No examinados } & \multicolumn{3}{|c|}{ No de casos } & \multicolumn{3}{|c|}{ Prevalência } & \multirow{2}{*}{$\mathbf{p}^{*}$} \\
\hline & $\mathrm{T}^{*}$ & $\mathbf{R}^{\dagger}$ & $\mathrm{U}^{\ddagger}$ & $\mathbf{T}$ & $\mathbf{R}$ & $\mathbf{U}$ & $\mathrm{T}$ & $\mathbf{R}$ & $\mathbf{U}$ & \\
\hline Agrestina & 3.543 & 994 & 2.549 & 65 & 14 & 51 & 1,8 & 1,4 & 2 & 0,24 \\
\hline Arcoverde & 8.651 & 1.199 & 7.452 & 244 & 56 & 188 & 2,8 & 4,7 & 2,5 & $<0,01$ \\
\hline Bodocó & 5.934 & 3.730 & 2.204 & 92 & 65 & 27 & 1,6 & 1,7 & 1,2 & 0,12 \\
\hline Betânia & 1.880 & 1.492 & 388 & 23 & 18 & 5 & 1,2 & 1,2 & 1,3 & 0,90 \\
\hline Canhotinho & 3.490 & 1.585 & 1.905 & 89 & 38 & 51 & 2,6 & 2,4 & 2,7 & 0,60 \\
\hline Cumaru & 2.490 & 877 & 1.613 & 111 & 52 & 59 & 4,5 & 6 & 3,7 & $<0,01$ \\
\hline Cupira & 3.629 & 653 & 2.976 & 65 & 22 & 43 & 1,8 & 3,4 & 1,4 & $<0,01$ \\
\hline Jurema & 977 & 433 & 544 & 14 & 7 & 7 & 1,4 & 1,6 & 1,3 & 0,66 \\
\hline Lajedo & 4.037 & 1.174 & 2.863 & 132 & 39 & 93 & 3,3 & 3,3 & 3,2 & 0,86 \\
\hline Moreno & 5.001 & 1.059 & 3.942 & 65 & 4 & 61 & 1,3 & 0,4 & 1,5 & $<0,01$ \\
\hline Palmares & 5.638 & 2.103 & 3.535 & 195 & 72 & 123 & 3,4 & 3,4 & 3,5 & 0,87 \\
\hline Pesqueira & 5.458 & 1.590 & 3.868 & 59 & 23 & 36 & 1,0 & 1,4 & 0,9 & 0,11 \\
\hline Poção & 2.175 & 509 & 1666 & 74 & 12 & 62 & 3,4 & 2,4 & 3,7 & 0,14 \\
\hline Saloá & 2.504 & 970 & 1.534 & 118 & 41 & 77 & 4,7 & 4,2 & 5 & 0,36 \\
\hline Sertânia & 3.494 & 1.129 & 2.365 & 50 & 12 & 38 & 1,7 & 1,1 & 1,6 & 0,20 \\
\hline São Joaquim do Monte & 2.447 & 1.052 & 1.395 & 21 & 12 & 9 & 0,8 & 1,1 & 0,6 & 0,12 \\
\hline Vertentes & 2.976 & 1.029 & 1.947 & 39 & 9 & 30 & 1,3 & 0,9 & 1,5 & 0,15 \\
\hline \multicolumn{11}{|l|}{ Barra de } \\
\hline Guabiraba & 1.905 & 70 & 1835 & 173 & 9 & 164 & 9,1 & 12,9 & 8,9 & 0,25 \\
\hline Bonito & 5.815 & 2.050 & 3.765 & 326 & 129 & 197 & 5,6 & 6,3 & 5,2 & 0,08 \\
\hline Ferreiros & 1.396 & 240 & 1.156 & 87 & 7 & 80 & 6,2 & 2,9 & 6,9 & 0,02 \\
\hline Iguaraci & 2.022 & 540 & 1.482 & 126 & 35 & 91 & 6,2 & 6,5 & 6,1 & 0,73 \\
\hline Paranatama & 2.659 & 1.643 & 1.016 & 203 & 117 & 86 & 7,6 & 7,1 & 8,5 & 0,20 \\
\hline Total & 78.118 & 26.120 & 51.998 & 2.306 & 793 & 1.578 & 2,95 & 3,03 & 3,03 & 0,98 \\
\hline
\end{tabular}

Fonte: Elaborado pela autora a partir dos dados da Secretaria Estadual de Saúde de Pernambuco, Programa SANAR (2011-2012)

Nota: T: total; ${ }^{\dagger} \mathrm{R}$ : rural; ${ }^{*} \mathrm{U}$ : urbano; ${ }^{*}$ Diferença entre urbana e rural

Tabela 2. Média, desvio-padrão e valor de $\mathrm{P}$ dos indicadores socioeconômicos, demográficos e saúde dos 79 municípios investigados para o tracoma, estratificados de acordo com o nível de prioridade para o controle do tracoma, em Pernambuco, Brasil, 2006/2012

\begin{tabular}{|c|c|c|c|}
\hline Indicadores & $\begin{array}{l}22 \text { Municípios } \\
\text { prioritários } \\
\text { Média DP }\end{array}$ & $\begin{array}{l}57 \text { Municípios não } \\
\text { prioritários } \\
\text { Média DP }\end{array}$ & P-valor \\
\hline Densidade demográfica & $99,79 \pm 74,54$ & $190,23 \pm 412,53$ & 0,84 \\
\hline PIB per capita & $5702,17 \pm 860,41$ & $9177,11 \pm 14987,47$ & 0,25 \\
\hline Índice Gini & $0,52 \pm 0,03$ & $0,52 \pm 0,45$ & 0,87 \\
\hline Taxa de frequência ao ensino pré-escolar & $53,33 \pm 13,06$ & $51,26 \pm 12,53$ & 0,73 \\
\hline Taxa de frequência ao ensino fundamental & $92,63 \pm 2,19$ & $91,63 \pm 2,57$ & 0,17 \\
\hline Taxa de frequência ao ensino médio & $29,38 \pm 5,64$ & $29,47 \pm 8,06$ & 0,89 \\
\hline Percentual de crianças extremamente pobres & $30,31 \pm 9,52$ & $26,58 \pm 13,10$ & 0,16 \\
\hline Percentual da população com domicílios com coleta lixo & $95,45 \pm 4,18$ & $92,85 \pm 6,33$ & 0,07 \\
\hline $\begin{array}{l}\text { Percentual da população com domicílios com abastecimento de água e } \\
\text { esgotamento sanitário inadequados }\end{array}$ & $17,89 \pm 12,28$ & $15,59 \pm 10,22$ & 0,47 \\
\hline Percentual da população em domicílios com banheiro e água encanada & $64,59 \pm 17,70$ & $65,40 \pm 18,99$ & 0,82 \\
\hline $\begin{array}{l}\text { Percentual da população em domicílios com densidade maior que } \\
\text { duas pessoas por dormitório }\end{array}$ & $30,22 \pm 5,01$ & $33,86 \pm 6,99$ & 0,03 \\
\hline Cobertura da Estratégia Saúde da Família & $88,20 \pm 15,75$ & $80,50 \pm 23,82$ & 0,26 \\
\hline
\end{tabular}

Fonte: Elaborado pela autora

Nota: DP: desvio-padrão; PIB: produto interno bruto 
Tabela 3. Matriz de correlação entre os indicadores socioeconômicos, demográficos e de saúde e a prevalência de tracoma dos 22 municípios prioritários para o tracoma, em Pernambuco, Brasil, 2012

\begin{tabular}{|c|c|c|}
\hline \multirow{2}{*}{$\begin{array}{c}\text { Indicadores } \\
\mathbf{r} \text { (Pearson) }\end{array}$} & \multicolumn{2}{|c|}{ Prevalência tracoma } \\
\hline & \multicolumn{2}{|c|}{ p-valor } \\
\hline Densidade demográfica & $-0,122$ & 0,590 \\
\hline População de 1 a 15 anos & $-0,351$ & 0,109 \\
\hline${ }^{\star}$ PIB per capita & $-0,138$ & 0,541 \\
\hline Índice Gini & $-0,008$ & 0,973 \\
\hline Taxa de frequência ao ensino pré-escolar & 0,272 & 0,221 \\
\hline Taxa de frequência ao ensino fundamental & 0,173 & 0,441 \\
\hline Taxa de frequência ao ensino médio & $-0,075$ & 0,741 \\
\hline Percentual de crianças extremamente pobres & $-0,031$ & 0,891 \\
\hline Percentual da população em domicílios com coleta lixo & 0,173 & 0,440 \\
\hline $\begin{array}{l}\text { Percentual da população em domicílios com abastecimento de água e esgotamento } \\
\text { sanitário inadequados }\end{array}$ & 0,223 & 0,318 \\
\hline Percentual da população em domicílios com banheiro e água encanada & $-0,062$ & 0,783 \\
\hline $\begin{array}{l}\text { Percentual da população em domicílios com densidade maior que duas pessoas por } \\
\text { dormitório }\end{array}$ & 0,155 & 0,491 \\
\hline Cobertura da Estratégia Saúde da Família & 0,117 & 0,603 \\
\hline
\end{tabular}

Fonte: Elaborado pela autora

Nota: ${ }^{\star}$ PIB: produto interno bruto

Baseando-se apenas nas prevalências pontuais dos 79 municípios desse inquérito, foram escolhidos os 22 municípios prioritários para o controle da doença dentro das iniciativas do Programa SANAR em 2011 e 2012. No entanto, ao analisar os IC para as prevalências desses 79 municípios, observa-se que outros 43 apresentavam probabilidade de ter prevalência maior que 5\%.

A não inclusão desses 43 municípios na agenda de ações para o enfrentamento do tracoma pode estar contribuindo para a manutenção de transmissão da doença no Estado, o que vai na contramão das metas de eliminação do tracoma como causa de cegueira no Brasil, as quais têm como estratégia de ação primordial a intensificação das práticas de busca ativa de casos de tracoma em sua forma inflamatória transmissível (TF/TI). Alguns fatores podem possibilitar a manutenção da cadeia de transmissão do tracoma, como a presença de crianças com formas de infecção ativa, mesmo em locais de baixa prevalência, constituindo-se, assim, fonte de infecção $0^{11}$.

Considerando as prevalências identificadas em 2012 nos 22 municípios prioritários, e seus IC, percebe-se que essas estimativas são mais precisas. Porém, se for levado em consideração que, em 2006, foi realizado o tratamento em todos os casos, chama a atenção o fato de que, ao compararem-se os IC das prevalências dos dois inquéritos escolares, não é possível, na grande maioria dos municípios, apontar redução estatisticamente significante da prevalência da doença. Esses achados, de certa forma, servem de reflexão para o que revela alguns estudos, quando destacam que as intervenções de curta duração, e de uma só vez, não dão conta da redução das altas prevalências do tracoma ${ }^{12,13}$.

As ações de educação em saúde e uma vigilância ativa se constituem em uma importante estratégia para o controle do tracoma, buscando a conscientização da população sobre a necessidade e adoção de hábitos de higiene e a importância do uso individual de objetos pessoais, como toalhas, fronhas, lençóis, entre outros ${ }^{14}$, aliando-se naturalmente a iniciativas para melhoria do saneamento e do fornecimento contínuo de água de boa qualidade.

As práticas educativas em saúde devem ser baseadas em ações que pressupõem a compreensão do outro como sujeito, detentor de um determinado conhecimento, respeitando o universo cultural dos participantes, o caráter histórico dos determinantes sociais, políticos e econômicos do processo saúde-doença e apresentadas como práticas dialógicas.

É importante destacar que, além de contribuir para a melhoria das condições de vida e saúde da população, devem ainda estimular a reflexão crítica das causas dos seus problemas, bem como das ações necessárias para sua resolução ${ }^{15,16}$.

Os dois grupos de municípios avaliados no presente estudo foram selecionados pelo valor do IDH-M (menor que a média nacional). Essa referência, por si só, tornou-os relativamente homogêneos no que diz respeito aos indicadores socioeconômicos, demográficos e de saúde, o que não permitiu identificar determinantes das diferenças de prevalência do tracoma. Além disso, o reduzido número de examinados no primeiro inquérito inviabilizou comparações com os resultados do inquérito realizado em 2012.

Diante desse cenário, mais uma vez questiona-se a não realização de um inquérito de base populacional para a doença a fim de se eleger, com bases técnicas adequadas, o conjunto de municípios a serem incluídos em uma agenda de prioridades.

Dos 22 municípios avaliados no inquérito de 2012, cinco apresentaram prevalências estatisticamente maiores que 
5\% - o município de Barra de Guabiraba teve a maior prevalência, com 9,1\% (IC:7,8-10,4\%), quando no inquérito anterior tinha apresentado prevalência de 7,1\% (IC:2,3-16,3\%).

Diante dos valores apresentados no IC do inquérito de 2006, não se pode afirmar que houve um aumento da prevalência no período entre os inquéritos escolares, mas pode-se concluir que o tracoma é um problema de saúde pública nesse município e, portanto, deve-se avaliar a necessidade de seguir a recomendação de tratamento em massa e outras medidas de controle preconizadas pela OMS e $\mathrm{MS}^{5,8}$.

Considerando as diferenças de características que apresentam as zonas rural e urbana dos municípios avaliados, no que tange às prevalências de tracoma, apenas em 5 municípios dos 22 avaliados foram encontradas diferenças estatisticamente significantes entre essas duas áreas, assim como em outros estudos com escolares, provavelmente relacionadas às precárias condições socioeconômicas muito frequentes na zona rural ${ }^{4,9}$.

Mesmo sem ter apresentado significância estatística entre as zonas rural e urbana, o município de Barra de Guabiraba destacou-se dos demais por ter apresentado na zona rural uma prevalência de 12,9\% (IC:6-23\%), mesmo apresentando o IC alargado, o que indica a possibilidade de que nessa área do município a prevalência seja maior que $10 \%$. Em relação à associação dos indicadores socioeconômicos, demográficos e de saúde selecionados neste estudo, não foi identificada correlação deles com a prevalência de tracoma, ou seja, a transmissão não pode ser explicada em âmbito ecológico nesse conjunto de 22 municípios, apontando uma homogeneidade socioambiental entre esses municípios, de maneira que não se justificaria o emprego de métodos multivariados de análise nesse caso. Em um inquérito epidemiológico realizado em crianças da $1^{\text {a }}$ a $4^{\text {a }}$ séries do ensino fundamental de todas as escolas públicas de Botucatu, no Estado de São Paulo, também não foram explicadas as características epidemiológicas do tracoma por meio dos indicadores sociodemográficos considerados ${ }^{17}$.

Já em outro estudo realizado com escolares em municípios do Amapá, na análise de correlação para medir a associação entre a prevalência do tracoma e as variáveis socioeconômicas dos municípios, com base no ano 2000, identificou-se moderada e positiva associação entre a prevalência do tracoma e o percentual de pessoas que viviam em domicílios com água encanada ${ }^{4}$.

Como o tracoma é uma doença que está relacionada com os níveis socioeconômicos da população, o MS orienta que as ações de controle da doença sejam desenvolvidas com a integração da atenção básica. Para isso, os profissionais devem ser capacitados para atender a essas demandas, inclusive identificando as vulnerabilidades da população a ser atendida, facilitando a compressão dos fenômenos resultantes do entrecruzamento de comportamento e vivências individuais, subjetivas, condições sociais, políticas e culturais, em conjunto com as ações de saúde de caráter permanente voltadas para a prevenção e controle dos $\operatorname{agravos}^{18}$.

\section{CONCLUSÃO}

O presente estudo identificou que no inquérito escolar nacional do tracoma, realizado em 2006 em Pernambuco, não foram selecionados todos os municípios com IDH-M abaixo da média nacional. Os IC das prevalências identificadas nesse inquérito se mostraram bastante amplos e evidenciaram uma imprecisão nos resultados, implicando em seleção enviesada dos 22 municípios considerados prioritários para o tracoma pelo Programa SANAR. Também não se pode comprovar a real diminuição da prevalência do tracoma na maioria dos municípios prioritários, podendo-se afirmar isso em apenas 5 dentre os 22 municípios selecionados. Diante desse contexto, recomenda-se que investigações de base populacionais sejam executadas acerca do tracoma no Estado e que as ações de controle sejam repensadas, já que o formato das investigações ainda é baseado em campanhas com ações pontuais, sem o desenvolvimento de outras mais contínuas e efetivas, reduzindo as formas ativas da doença, para assim alcançar a meta de eliminação do tracoma como causa de cegueira.

\section{REFERÊNCIAS}

1. Brasil. Ministério da Saúde. Secretaria de Vigilância em Saúde. Manual de vigilância do tracoma e sua eliminação como causa de cegueira. 2. ed. Brasília; 2014.

2. Luna EJA, Medina NH, Oliveira MB, Barros OM, Vranjac A, Melles HHB, et al. Epidemiology of trachoma in Bebedouro, State of São Paulo, Brazil: Prevalence and risk factors. Int J Epidemiol. 1992;21(1):169-77. PMid:1544750. http://dx.doi.org/10.1093/ije/21.1.169.

3. Reilly LA, Favacho J, Garcez LM, Courtenay O. Preliminary evidence that synanthropic flies contribute to the transmission of trachoma: causing
Chlamydia trachomatis in Latin America. Cad Saude Publica. 2007;23(7):16828. PMid:17572818. http://dx.doi.org/10.1590/S0102-311X2007000700020.

4. Pinto ICS. Fatores epidemiológicos associados à prevalência do tracoma no Estado do Amapá [dissertação]. Rio de Janeiro: Fundação Oswaldo Cruz, Escola Nacional de Saúde Pública; 2011.

5. World Health Organization. London Scholl of Hygiene \& Tropical Medicine \& International Trachoma Initiative. Trachoma control: a guide for programme managers. Geneva: WHO; 2006. 
6. World Health Organization, Pan American Health Organization. Key facts on Neglected Infectious Diseases Blinding Trachoma. PAHO/WHO; 2014 [citado em 2014 set 20]. Disponível em: http://www.paho.org/hq/index. php?option=com_docman\&task $=$ doc_download\&Itemid $=\&$ gid $=24738 \&$ lang=en_Suplemento.pdf.

7. World Health Organization. Alliance for the Global Elimination of Blinding Trachoma by 2020: report of the 2nd Global Scientific Meeting on Trachoma. Geneva: WHO; 2003.

8. Brasil. Ministério da Saúde. Secretaria de Vigilância em Saúde. Plano integrado de ações estratégicas de eliminação da hanseníase, filariose, esquistossomose e oncocercose como problema de saúde pública, tracoma como causa de cegueira e controle das geohelmintíases: plano de ação 2011-2015. Brasília; 2012.

9. Lopes MFC, Luna EJA, Cardoso MRA, Medina NH, Freitas HSA, Koizumi IK, et al. Prevalência de tracoma entre escolares brasileiros. Rev Saude Publica. 2013;47(3):451-9. PMid:24346557. http://dx.doi.org/10.1590/ S0034-8910.2013047003428.

10. Brasil. Secretaria de Saúde do Estado de Pernambuco. Secretaria Executiva de Vigilância em Saúde. Programa de enfrentamento das doenças negligenciadas no estado de Pernambuco - 2011/2014. Recife: SES/PE; 2013.

11. Brasil. Ministério da Saúde. Secretaria de Atenção à Saúde. Vigilância em Saúde: Dengue, Esquistossomose, Hanseníase, Malária, Tracoma e Tuberculose. 2. ed. Brasília: 2008.

12. West SK, Munoz B, Mkocha H, Gaydos CA, Quinn TC. Number of years of annual mass treatment with azithromycin needed to control trachoma in hyper-endemic communities in Tanzania. J Infect Dis. 2011;204(2):268-73. PMid:21673038. http://dx.doi.org/10.1093/infdis/jir257.

13. Lavett DK, Lansingh VC, Carter MJ, Eckert KA, Silva JC. Will the SAFE Strategy be sufficient to eliminate trachoma by 2020 ? Puzzlements and Possible Solutions. The Scientific World Journal. 2013;648106:1-18.

14. Koizumi IK, Medina NH, D’amaral RKK, Morimoto WTM, Caligaris LSA, Chinen N, et al. Prevalência do tracoma em pré-escolares e escolares no Município de São Paulo. Rev Saude Publica. 2005;39(6):937-42. PMid:16341404. http://dx.doi.org/10.1590/S0034-89102005000600011.

15. Acioli S. A prática educativa como expressão do cuidado em Saúde Pública. Rev Bras Enferm. 2008;61(1):117-21. PMid:18578320. http://dx.doi. org/10.1590/S0034-71672008000100019.

16. Maciel MED. Educação em saúde: conceitos e propósitos. Cogitare Enferm. 2009;14(4):773-6. http://dx.doi.org/10.5380/ce.v14i4.16399.

17. Schellini SA, Lavezzo MM, Ferraz LB, Olbrich No J, Medina NH, Padovano CR. Prevalência e localização espacial dos casos de tracoma detectados em escolares de Botucatu, São Paulo - Brasil. Arq Bras Oftalmol. 2010;73(4):35862. PMid:20944941. http://dx.doi.org/10.1590/S0004-27492010000400012.

18. Nichiata LYI, Bertolozzi MR, Takahashi RF, Fracolli LA. The use of the "vulnerability" concept in the nursing area. Rev Latino-am Enfermagem. 2008;16(5):923-8. PMid:19061032. http://dx.doi.org/10.1590/S010411692008000500020 .

Recebido em: Maio 19, 2016 Aprovado em: Out. 10, 2016 